\title{
Atrial Activity Enhancement by Blind Sparse Sequential Separation
}

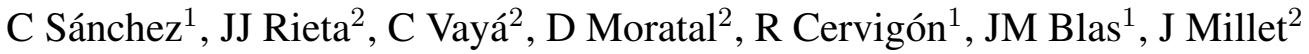 \\ ${ }^{1}$ Innovation in Bioengineering, Castilla-La Mancha University, Cuenca, Spain \\ ${ }^{2}$ Bioengineering, Electronics and Telemedicine, Valencia University of Technology, Spain
}

\begin{abstract}
Blind Source Separation (BSS) has been probed as one of the most effective techniques for the atrial activity ( $A A)$ extraction in supraventricular tachyarrhythmia episodes like atrial fibrillation $(A F)$. In these situations, the registered episodes with only a few leads are noisy and timevarying and previous stages for sparse separation have been demonstrated as necessary. Including wavelet transform de-noising and natural gradient algorithm for the BSS system can improve the extraction quality with low computational load. Synthetic signals have been used to test the proposed technique in different noisy cases. The obtained cross-correlation coefficients with sparse sequential separation between the extracted signal and the original ones exceed the $94 \%$ in contrast with the obtained results using standalone BSS method. The easy and fast implementation and the minimum required reference recordings of the same ECG are some of the main advantages of this technique and make the application in real time systems possible.
\end{abstract}

\section{Introduction}

Atrial fibrillation (AF) is one of the most common arrhythmias and causes the highest number of admissions in casualty department of hospitals.

The prevalence of this supraventricular tachyarrhythmia is estimated at $0.4 \%$ of the general population and the median age of the AF patients is 75 years. The incidence increases with the age [1] and is slightly more common in women $[2,3]$. About $1 \%$ of the people above 60 years suffer from AF. These numbers raise by $6 \%$ in people above 80. AF is relatively rare in the people above 20 years.

Symptoms associated with AF depend on several factors but most patients experience palpitations, chest pain, lightheadedness, presyncopes, dizziness, fatigue and dyspnea. Clearly, this arrhythmia may directly impact the quality of life. Nowadays, the treatment and analysis of AF is not completely satisfactory, and the high levels of morbidity, mortality and associated costs give rise to many scientific works and publications about this theme [4].
Blind Source Separation (BSS) has been probed as one of the most effective techniques for the atrial activity (AA) extraction in supraventricular tachyarrhythmia episodes like atrial fibrillation (AF) [5], [6]. Nevertheless, the reduced number of used reference signals (leads) and the presence of noise can decrease the efficiency of these methods.

In recent works, a wavelet stage of de-noising has been proved as a very efficient preprocessing technique which improves the performance of certain BSS applications [7], [8]. The definition of the observed mixtures in the wavelet domain reduces the gaussian features of these signals and speeds up the convergence.

\subsection{BSS principles}

$\mathrm{BSS}$, as a processing tool, is able to recover signals from a linear combination of these sources without any a priori knowledge [9]. The simplest model of BSS takes on the presence of $n$ statically independent signals and $n$ observed linear and instantaneous mixtures. In this work, the independence and nongaussianity of the atria and ventricle as signal sources is taken on. Recent works have studied the propagation mechanisms and uncoordinated atrial activation of the AF to demonstrate this assumption [10].

The Independent Component Analysis (ICA) based on higher order statistics is the most extended support for the different methodologies that solve the problem of BSS. The BSS model in its more compact form is given by

$$
\begin{gathered}
\mathbf{x}(t)=\sum_{j=1}^{n}\left[a_{i, j} \cdot s_{j}(t)\right] \\
\mathbf{x}(\mathbf{t})=\mathbf{A} \cdot \mathbf{s}(\mathbf{t})
\end{gathered}
$$

where, $\mathbf{s}(\mathbf{t})$ is a vector of $n \times 1$ columns which contains the estimated sources, $x(t)$ is the vector of the mixtures and $\mathbf{A}$ is the square mixing matrix. As BSS tries to recover $\mathbf{s}(\mathbf{t})$ from the observations, $\mathbf{x}(\mathbf{t})$ is necessary to estimate the matrix A. If the ICA methods can estimate the separation matrix, the independent sources can be expressed as follows 


$$
\hat{\mathbf{s}}(t)=\mathbf{y}(\mathbf{t})=\mathbf{B} \cdot \mathbf{x}(\mathbf{t})
$$

where $\hat{\mathbf{s}}(t)$ is the vector of estimated sources and $\mathbf{B}$ is the separation matrix that recovers the independent sources.

\subsection{Wavelet Transform principles}

Wavelet analysis is used to transform the signal under investigation into another representation that presents the signal information in a more useful form, joining spectral and temporal analysis [11].

Mathematically speaking, the Wavelet Transform (WT) is a convolution of the wavelet function $\psi_{a, b}$ (a dilated and displaced version of the "mother wavelet" $\psi$, where the parameters $a$ and $b$ indicate scale and translation, respectively) with the signal $x(t)$.

The discrete wavelet transform (DWT) results from discretizing scale and translation parameters. The definition of parameters as $a=2^{j}$ and $b=k \cdot 2^{j}$ leads to the dyadic DWT (DyWT), expressed as (4).

$$
\begin{gathered}
c(j, k)=\sum_{n \in Z}\left[x[n] \psi_{j, k}[n]\right] \\
\psi_{j, k}(n)=2^{-j / 2} \psi\left[2^{-j} n-k\right] \\
a=2^{j} \quad b=k 2^{j} \quad(j, k) \in Z^{2}
\end{gathered}
$$

\subsection{Database}

Synthetic sinus rhythm episodes obtained with the EcgSyn software from Physionet [12] have been used to test the proposed technique in different noisy cases (no noise, $10 \mathrm{~dB}$ and $5 \mathrm{~dB}$ signal to noise ratio (SNR)). $\mathrm{P}$ wave has been removed in all these episodes and a synthesized AF signal has been added using linear combination with randomly generated matrixes, according with the studies and references of several authors [13]. These registers have four leads and the synthesis process can be observed in Figures 1-2 and Equation (5),

$$
\mathbf{d}=\mathbf{M} \cdot \mathbf{s}_{\mathbf{y}}
$$

where, $\mathbf{d}=\left[D_{1}, D_{2}, D_{3}, D_{4}\right]$ is the vector of synthetic $\mathrm{AF}$ episodes, $\mathbf{S}_{\mathbf{y}}=\left[s_{1}, s_{2}\right]$ contains the simulated atrial and ventricular activities and $\mathbf{M}$ is the random combination matrix. The final configuration of the database is shown in Table 1.

Table 1. Registers in database

\begin{tabular}{cc}
\hline \hline Artificial Mixtures & Real registers \\
\hline 12 & 16 \\
\hline \hline
\end{tabular}
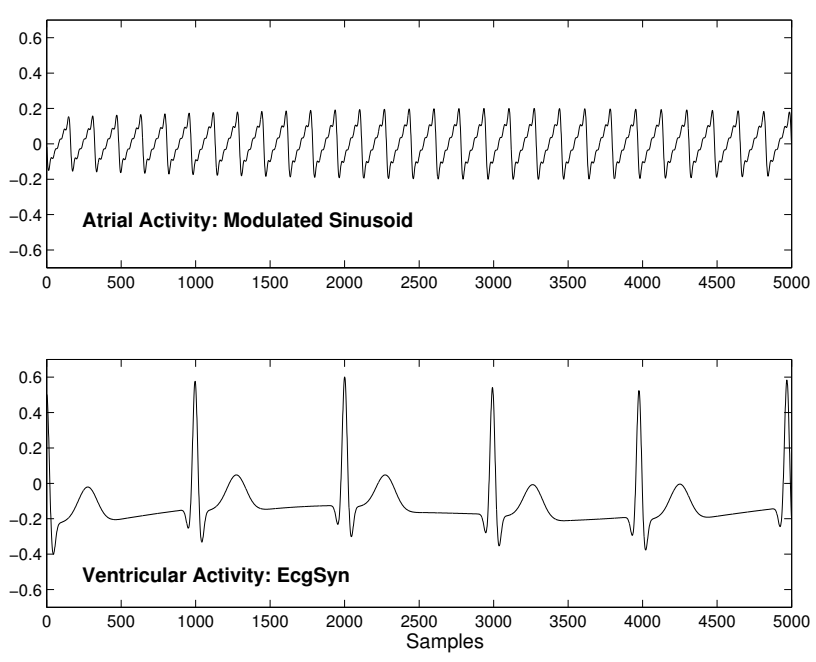

Figure 1. Original sources: atrial (Modulated Sinusoid) and ventricular activity (EcgSyn, $P$ wave removed).
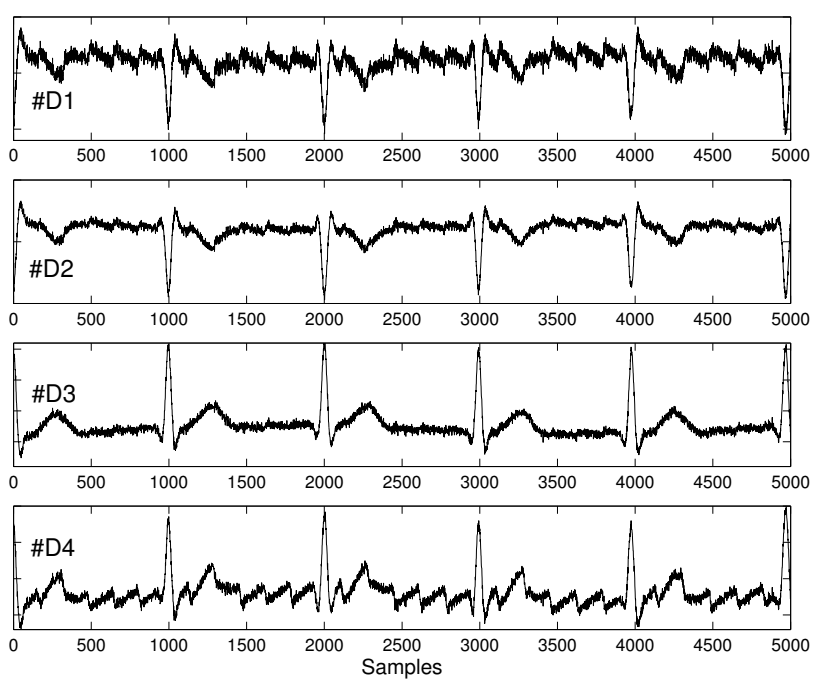

Figure 2. Example of Synthetic AF episode with Gaussian noise at $10 \mathrm{~dB}$ SNR (Four Leads).

\section{Methods}

Taking the classic definition of the BSS problem as a starting point, Equation (2), the wavelet coefficients of each signal can be expressed as:

$$
\begin{aligned}
& \mathbf{c}_{x}=\sum \mathbf{x}(t) \psi(t) \\
& \mathbf{c}_{s}=\sum \mathbf{s}(t) \psi(t)
\end{aligned}
$$

where $\mathbf{c}_{x}$ and $\mathbf{c}_{s}$ represent the wavelet coefficient vectors of the mixtures and the original sources respectively. Using a de-noising stage, some of these coefficients become zero and the decomposition can be considered as optimal. After 
that, a new formulation of the blind separation problem can be done:

$$
\mathbf{c}_{x}=\mathbf{A} \cdot \mathbf{c}_{s}
$$

These new mixtures present higher kurtosis values, becoming less Gaussian. According with (3), in the wavelet domain the estimated sources are expressed by:

$$
\hat{\mathbf{c}}_{s}=\mathbf{y}_{s}=\mathbf{B} \cdot \mathbf{c}_{x}
$$

Finally, the original sources are obtained with the inverse wavelet transform of the vectors defined by (9):

$$
\mathbf{s}(t)=\sum_{j} \sum_{k} \mathbf{c}_{s} \psi_{j, k}(t)
$$

The previous process can be applied in consecutive blocks of the input signal. These sequential approach decreases the computation time up to $85 \%$ and speeds up the convergence. The optimal length of the blocks is set to 2000 samples.

The function biorthogonal 4.4 and five levels of decomposition are the wavelet family and the configuration with the best performance respectively.

Spectral analysis has been used to identify the AA between the obtained signals. The signal with a principal frequency peak in the band of 5-8 Hz and higher spectral concentration in this range -as it is usual in an AF episodeis identified as AA.

\section{Results}

Both methods have been applied to the registers in Table 1 and the final results with their mean value and standard deviation have been presented in Table 2. The comparison between the obtained AA and the expected one can be done with the cross correlation coefficients in spectral (Corr-F) and temporal domain (Corr-T). Other additional parameters have been considered, for example, principal peak $(f p)$ and spectral energy concentration in the band of $5-8 \mathrm{~Hz}$ (named as $S C B P$ ). The high energy concentration in this band in respect of the total energy is typical in AF episodes that present a dominant peak in this range. An increase of this concentration involves a greater purity of the extracted AA and it is considered as quality extraction index. The Welch's averaged, modified periodogram method $(50 \%$ overlap, Hamming window, NFFT $=8192)$ has been used to estimate the power spectral density (PSD).

As can be observed in Table 2, the noise presence reduce the quality extraction clearly. In the case of noiseless registers the obtained correlation coefficients and spectral parameters are very similar in the considered methods but, when the signal to noise ratio decreases and the noise influence is more evident, the efficiency of Blind Sparse Separation is better in all aspects.
The sources identified as AA with the ICA separation method shows the presence of residual complexes, Figure 3, this fact explains the low levels of SCBP, Figure 4.

Table 2. Spectral parameters and correlation coefficients obtained with ICA and Blind Sparse Separation methods in different noisy cases.

\begin{tabular}{|c|c|c|}
\hline \hline No noise & ICA & Blind Sparse \\
\hline fp $(\mathrm{Hz})$ & $6.10 \pm 0.02$ & $6.10 \pm 0.01$ \\
SCBP & $0.75 \pm 0.10$ & $0.71 \pm 0.08$ \\
Corr-F & $0.99 \pm 0.06$ & $0.99 \pm 0.11$ \\
Coor-T & $0.99 \pm 0.14$ & $0.98 \pm 0.09$ \\
\hline \hline $10 \mathrm{~dB} \mathrm{SNR}$ & ICA & Blind Sparse \\
\hline fp(Hz) & $6.10 \pm 0.24$ & $6.10 \pm 0.12$ \\
SCBP & $0.55 \pm 0.15$ & $0.69 \pm 0.08$ \\
Corr-F & $0.85 \pm 0.06$ & $0.92 \pm 0.11$ \\
Coor-T & $0.79 \pm 0.14$ & $0.98 \pm 0.09$ \\
\hline \hline 5 dB SNR & ICA & Blind Sparse \\
\hline fp(Hz) & $6.10 \pm 0.51$ & $6.10 \pm 0.36$ \\
SCBP & $0.43 \pm 0.10$ & $0.66 \pm 0.08$ \\
Corr-F & $0.69 \pm 0.06$ & $0.92 \pm 0.11$ \\
Coor-T & $0.71 \pm 0.11$ & $0.95 \pm 0.09$ \\
\hline \hline
\end{tabular}

\section{Discussion and conclusions}

As the low pass filter used a preprocessing stage can impact the performance of ICA methods in the case of AA extraction from AF episodes, the Blind Sparse Separation method is presented as an alternative to these traditional de-noising systems.

The reduction of the gaussian features of the mixtures in the wavelet domain improves the convergence of the ICA algorithm. This increase of the computational speed makes the proposed method suitable for real time applications.

The positive results reported in this paper show that the Blind Sparse process should be applicable in arrhythmia detection and analysis, like paroxysmal atrial fibrillation, which have to be usually detected from Holter systems where the number of reference signals is reduced.

\section{Acknowledgements}

This work was partly funded by several research grants from Junta de Comunidades de Castilla-La Mancha (Ref: PAC-05-008-1) and Consellería de Empresa Universidad y Ciencia de la Generalitat Valenciana (Ref: IIARC0/2004/249). 


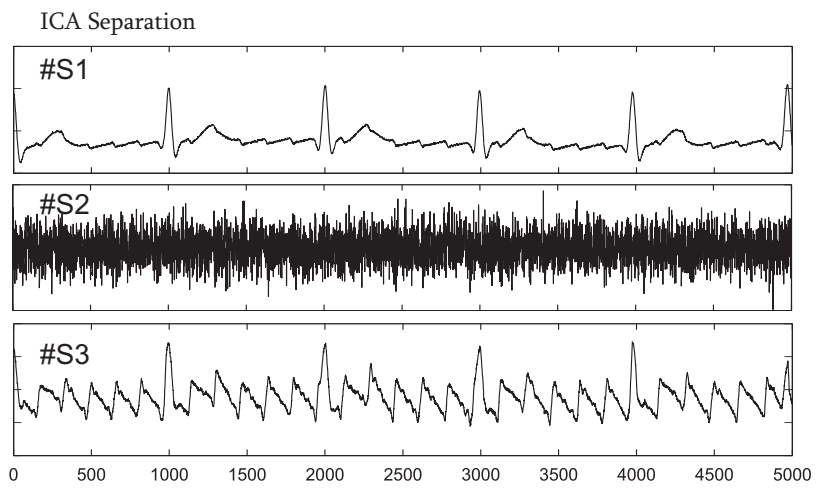

Blind Sparse Separation
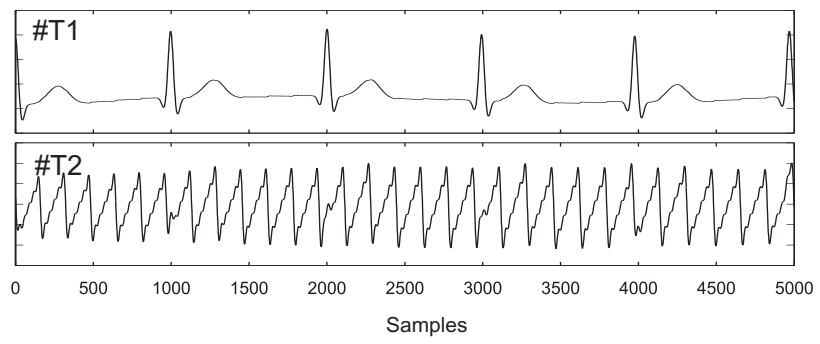

Figure 3. Example of estimated sources with ICA (S13) and Blind Sparse Separation (T1-2) in the case of $5 \mathrm{~dB}$ SNR.
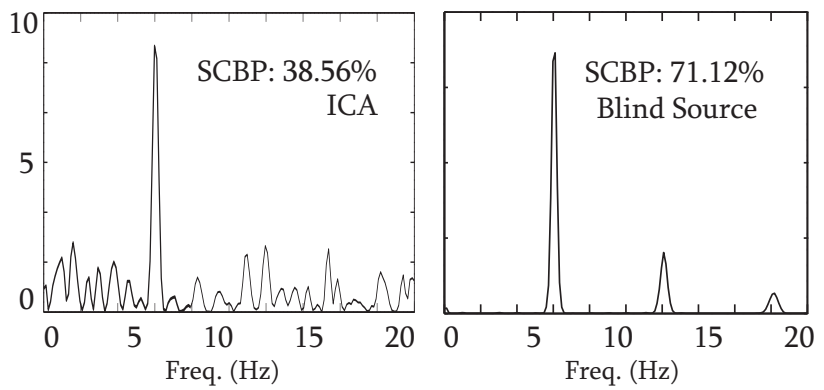

Figure 4. Example of Power Spectral Density of estimated sources with ICA and Blind Sparse Separation in the case of $5 \mathrm{~dB}$ SNR.

\section{References}

[1] Falk RH. Medical progress: Atrial fibrillation. New England Journal of Medicine 2001;344(14):1067-1078.

[2] Kannel WB, Abbott R, Savage DD, McNamara PM. Epidemiologic features of chronic atrial fibrillation: the Framingham study. New England Journal of Medicine 1982; 306:1018-1022.

[3] Furberg CD, Psaty BM, Manolio TA, Gardin JM, Smith VE, Rautaharju PM. Prevalence of atrial fibrillation in elderly subjects (the Cardiological Health Study ). Am J Cardiol 1994; 74:236-241.

[4] Fuster V, Ryden LE, Asinger RW, et al. ACC/AHA/ESC guidelines for the management of patients with atrial fibrillation: a report of the American College of Cardio-
logy/American Heart Association Task Force on practice guidelines and the European Society of Cardiology (committee to develop guidelines for the management of patients with atrial fibrillation). Journal of the American College of Cardiology 2001;38(4):1266i-1266lxx.

[5] Rieta JJ, Castells F, Sánchez C, Zarzoso V, Millet J. Atrial activity extraction for atrial fibrillation analysis using blind source separation. IEEE Trans Biomed Eng 2004; 51(7):1176-1186.

[6] Castells F, Rieta JJ, Millet J, Zarzoso V. Spatiotemporal blind source separation approach to atrial activity estimation in atril tachyarrhythmias. IEEE Trans Biomed Eng 2005;52(2):258-267.

[7] Jafari M, Chmabers J. Fetal electrocardiogram extraction by sequential source separation in the wavelet domain. IEEE Trans Biomed Eng 2005;52(3):390-400.

[8] Sánchez C, Rieta JJ, Castells F, Alcaraz R, Millet J. Wavelet blind separation: A new methodology for the analysis of atrial fibrillation from Holter recordings. In Computers in Cardiology, volume 31. Los Alamitos, CA: IEEE, 2004; 417-420.

[9] Cichocki A, Amari S. Adaptive Blind Signal and Image Processing: Learning Algorthms and Applications. John Wiley \& Sons, 2003. ISBN 0471-60791-6.

[10] Rieta JJ, Castells F, Sánchez C, Moratal-Pérez D, Millet J. Bioelectric model of atrial fibrillation: Applicability of blind source separation techniques for atrial activity estimation in atrial fibrillation episodes. In Computers in Cardiology, volume 30. Los Alamitos, CA: IEEE, 2003; 525-528.

[11] Addison PS. The Illustrated Wavelet Transform Handbook. Introductory Theory and Applications in Science, Engineering, Medicine and Finance. Institute of Physics Publishing, 2002. ISBN 0-7503-0692-0.

[12] McSharry P, Clifford G, Tarassenko L, Smith L. A dynamical model for generating synthetic electrocardiogram signals. IEEE Trans Biomed Eng March 2003;50(3):289-294.

[13] Stridh M, Sornmo L. Spatiotemporal QRST cancellation techniques for analysis of atrial fibrillation. IEEE Trans Biomed Eng 2001;48(1):105-111.

Address for correspondence:

César Sánchez Meléndez

EUP Cuenca, Universidad de Castilla-La Mancha Campus Universitario s/n, 16071 Cuenca. Spain cesar.sanchez@uclm.es 